\title{
Neuroprotective and Ameliorative Actions of Polyunsaturated Fatty Acids Against Neuronal Diseases - Evidence From Basic to Clinical Studies: Preface
}

\author{
Tomohito Hamazaki $^{1, *}$ and Michio Hashimoto ${ }^{2}$ \\ ${ }^{1}$ Department of Clinical Sciences, Institute of Natural Medicine, University of Toyama, \\ 2630 Sugitani, Toyama-city, Toyama 930-0194, Japan \\ ${ }^{2}$ Department of Environmental Physiology, Shimane University Faculty of Medicine, \\ 89-1 Enyacho, Izumo, Shimane 693-8501, Japan
}

Received March 25, 2011; Accepted March 28, 2011

Keywords: Alzheimer's disease, n-3 polyunsaturated fatty acid, behavior, hostility, neurogenesis

The brain is completely different from other tissues. One of the most distinct aspects of the brain is its abundance in membranes, which contain tremendous amounts of cholesterol and phospholipids with arachidonic and docosahexaenoic acids. Those fatty acids are sold as brain supplements. However, scientific evidence for the effects of those fatty acids on brain functions is not necessarily sufficient. A symposium was held to clarify the neuroprotective and ameliorative actions of polyunsaturated fatty acids (PUFA) at the 83rd Annual Meeting of The Japanese Pharmacological Society held in March 2010, in Osaka, Japan.

Four invited scientists discussed animal and human studies about the effects of PUFA on brain functions. $M$. Hashimoto showed the protective effects of n-3 PURA, especially docosahexaenoic acid (DHA), against Alzheimer's disease using a rat model and the DHAinduced inhibition of amyloid fibrillation and discussed the mechanism of the protective effect of DHA. T. Moriguchi showed the change in the emotional behavior as a brain function in n-3 PUFA-deficient mice, and discussed the relation between the fatty acid composition and the sensitivity of the mild stress (1). T. Hamazaki conducted a number of intervention trials with fish oil and discussed the beneficial effects of fish oil administration on hostility (2). T. Yamashima focused on the functions of a fatty acid receptor, GPR40 (G protein-coupled receptor 40), in the brain and proposed new links between PUFA, GPR40, and adult hippocampal neurogenesis.

Among these four presentations in the symposium, we show two topics with their detailed discussions and proposals here in this Forum Minireview series $(3,4)$. We hope that these inspire pharmacology researchers to find new facets of brain research.

\section{References}

1 Moriguchi T. The emotional behavior and brain fatty acid composition in the $\mathrm{n}-3$ fatty acid deficient mice. J Pharmacol Sci. 2010;112 Suppl 1:30P.

2 Hamazaki T, Itomura M, Hamazaki K, Watari M, Fujita N. n-3 fatty acids and control of hostility. J Pharmacol Sci. 2010;112 Suppl 1:31P.

3 Hashimoto M, Hossain S. Neuroprotective and ameliorative actions of polyunsaturated fatty acids against neuronal diseases: Beneficial effect of docosahexaenoic acid on cognitive decline in Alzheimer's disease. J Pharmacol Sci. 2011;116:150-162.

4 Boneva NB, Kikuchi M, Minabe Y, Yamashima T. Neuroprotective and ameliorative actions of polyunsaturated fatty acids against neuronal diseases: Implication of fatty acid-binding proteins (FABP) and G protein-coupled receptor 40 (GPR40) in adult neurogenesis. J Pharmacol Sci. 2011;116:163-172.

*Corresponding author. hamazaki@inm.u-toyama.ac.jp Published online in J-STAGE on May 21, 2011 (in advance) doi: 10.1254/jphs.11R02FM 